\title{
Rapid directional alignment of velocity and magnetic field in magnetohydrodynamic turbulence
}

\author{
W. H. Matthaeus ${ }^{1}$, A. Pouquet ${ }^{2}$, P. D. Mininni ${ }^{2,3}$, P. Dmitruk ${ }^{1}$, and B. Breech ${ }^{1}$ \\ 1 Bartol Research Institute and Department of Physics and Astronomy, \\ University of Delaware, Newark DE 18716, U.S.A. \\ 2 NCAR, P.O. Box 3000, Boulder, Colorado 80307-3000, U.S.A. \\ 3 Departamento de Física, Facultad de Ciencias Exactas y Naturales, \\ Universidad de Buenos Aires, Ciudad Universitaria, 1428 Buenos Aires, Argentina.
}

(Dated: October 26, 2018)

\begin{abstract}
We show that local directional alignment of the velocity and magnetic field fluctuations occurs rapidly in magnetohydrodynamics for a variety of parameters. This is observed both in direct numerical simulations and in solar wind data. The phenomenon is due to an alignment between the magnetic field and either pressure gradients or shear-associated kinetic energy gradients. A similar alignment, of velocity and vorticity, occurs in the Navier Stokes fluid case. This may be the most rapid and robust relaxation process in turbulent flows, and leads to a local weakening of the nonlinear terms in the small scale vorticity and current structures where alignment takes place.
\end{abstract}

In magnetohydrodynamic (MHD) turbulence, the fluctuating magnetic field $\mathbf{b}$ and velocity fluctuation $\mathbf{v}$ enter on nearly equal footing. One consequence is that the nonlinear MHD equations are in effect linearized when the fluctuation components of the magnetic field (in Alfvén speed units) are everywhere equal (or opposite) to the velocity field. Such "Alfvénic" states have long been thought to be favored in relaxation processes [1]. Near Alfvénic states are observed in the solar wind plasma [2], mostly in the inner heliosphere [3]. Global evolution towards the Alfvénic state, or "dynamic alignment," [4], when it occurs, requires many nonlinear eddy turnover times. Here we describe a related, more rapid relaxation process, in which local, directional near-alignment of $\mathbf{v}$ and $\mathbf{b}$ emerges in less than one turnover time, for a wide variety of turbulence parameters. This process need not be associated with global alignment, but rather occurs independently in numerous localized patches.

Dynamic alignment competes with other MHD relaxation processes as shown in simulations [6] where, for some parameters, it does not occur, or is incompletely realized. Solar wind observations also show that the degree of Alfvénicity tends to decrease with increasing heliocentric distance, in apparent contradiction to the dynamic alignment principle. There are suggestions that directional alignment (a necessary condition for global dynamic alignment) may be more ubiquitous. When MHD relaxation is described by a constrained energy minimization principle [6], cross helicity (Alfvénicity) and a magnetic invariant (helicity in three dimensions - 3D; or mean square flux function in two dimensions - 2D) are held constant, while energy is minimized. The emergent Euler-Lagrange equations predict final states, in both 2D and $3 \mathrm{D}$, and for all parameters, in which $\mathbf{v}$ and $\mathbf{b}$ are directionally aligned or anti-aligned. This theory is reasonably well confirmed by direct numerical simulations. Evidently, in the long time limit for decaying MHD turbulence, pointwise directional alignment is obtained more easily than is the global Alfvénic state. Below we show, using MHD numerical experiments, that local directional alignment is even more robust, occurs more rapidly, and appears locally, in patches. The distribution of alignment angle in the solar wind is shown to be consistent with this picture. This rapid relaxation can be understood by an elementary examination of the MHD equations. While these features appear not to have been fully recognized previously, the situation is in fact analogous to the local emergence of Beltrami flows [7] in hydrodynamics.

MHD and Alfvénic states. In familiar dimensionless ("Alfvénic") units, the equations of incompressible MHD are

$$
\begin{aligned}
& \frac{\partial \mathbf{v}}{\partial t}+\mathbf{v} \cdot \nabla \mathbf{v}=-\nabla \mathcal{P}+\mathbf{j} \times \mathbf{b}+\nu \nabla^{2} \mathbf{v} \\
& \frac{\partial \mathbf{b}}{\partial t}+\mathbf{v} \cdot \nabla \mathbf{b}=\mathbf{b} \cdot \nabla \mathbf{v}+\eta \nabla^{2} \mathbf{b},
\end{aligned}
$$

with $\nabla \cdot \mathbf{v}=\nabla \cdot \mathbf{b}=0$. Here, $\mathbf{v}$ is the velocity field, and $\mathbf{b}$ is the magnetic field, related to the electric current density $\mathbf{j}$ by $\nabla \times \mathbf{b}=\mathbf{j} ; \mathcal{P}$ is the pressure. The viscosity $\nu$ and magnetic diffusivity $\eta$ define mechanical and magnetic Reynolds numbers respectively as $R_{V}=L U / \nu$ and $R_{M}=L U / \eta$. Here $U=\left\langle|\mathbf{v}|^{2}\right\rangle^{1 / 2}$, with $\langle\ldots\rangle$ denoting a spatial average, and $L$ is a length scale associated with the large-scale flow (integral length scale). The total energy $E=E_{v}+E_{b}=\left\langle|\mathbf{v}|^{2}+|\mathbf{b}|^{2}\right\rangle / 2$, the cross helicity $H_{c}=\langle\mathbf{v} \cdot \mathbf{b}\rangle$, and the magnetic helicity $H_{m}=\langle\mathbf{a} \cdot \mathbf{b}\rangle$ are ideal $(\nu=\eta=0)$ invariants in $3 \mathrm{D}$. Here $\mathbf{b}=\nabla \times \mathbf{a}$. Dimensionless measures of the helicities are $\sigma_{c}=2 H_{c} / E$ and $\sigma_{m}=\left(E_{L}-E_{R}\right) / E_{b}$, where $E_{L}$ and $E_{R}$ are magnetic energy in left- and right-handed magnetic polarizations, respectively.

Simulations. We consider several sets of simulations (see Table I), in which the MHD equations are integrated in a spatially periodic domain of side $2 \pi$, using a second order Runge-Kutta method, and either 2/3-rule dealiased [8], or non-dealiased pseudospectral methods. All runs freely decay in time, with no external forcing.

The type labelled RAN are $128^{3}$ incompressible runs, with random broadband initial conditions. Four cases are distinguished by their values of $\sigma_{m}$ and $\sigma_{c}$, spanning 
TABLE I: Parameters in the MHD simulations shown in the figures. RAN, OT, and $\mathrm{ABC}$ are described in the text. $N$ is the resolution, $\nu$ and $\eta$ are respectively the kinematic viscosity and magnetic diffusivity, and $\sigma_{c}$ and $\sigma_{m}$ reffer to the normalized cross and magnetic helicities defined in the text. For the $2 \mathrm{D}$ run, $\sigma_{m}$ is based on the mean-square flux function.

\begin{tabular}{ccccc}
\hline \hline Run & $N^{3}$ & $\nu=\eta$ & $\sigma_{c}$ & $\sigma_{m}$ \\
\hline RAN1 & $128^{3}$ & $2.5 \times 10^{-3}$ & 0 & 0 \\
RAN2 & $128^{3}$ & $2.5 \times 10^{-3}$ & 0.5 & 0 \\
RAN3 & $128^{3}$ & $2.5 \times 10^{-3}$ & 0 & 0.5 \\
RAN4 & $128^{3}$ & $2.5 \times 10^{-3}$ & 0.5 & 0.5 \\
\hline OT1 & $128^{3}$ & $5 \times 10^{-3}$ & 0.4 & 0 \\
OT2 & $256^{3}$ & $1.5 \times 10^{-3}$ & 0.4 & 0 \\
OT3 & $512^{3}$ & $7.5 \times 10^{-4}$ & 0.4 & 0 \\
\hline ABC1 & $128^{3}$ & $3 \times 10^{-3}$ & 0 & 0.5 \\
ABC2 & $256^{3}$ & $1.25 \times 10^{-3}$ & 0 & 0.5 \\
ABC3 & $512^{3}$ & $6 \times 10^{-4}$ & 0 & 0.5 \\
ABC4 & $1536^{3}$ & $2 \times 10^{-4}$ & 0 & 0.5 \\
\hline 2D & $1024^{2}$ & $2.5 \times 10^{-4}$ & 0 & 0 \\
\hline \hline
\end{tabular}

a range of possibilities for relaxation starting from a fully random state.

We also employ two other types of initial conditions in which helicities are controlled and the fields are more ordered. OT runs are a generalization of the 2D OrszagTang (OT) vortex [9], a standard large scale initial condition for MHD turbulence. In our OT case, initially energies $E_{v}=E_{b}=2, \sigma_{c} \approx 0.4$, and $\sigma_{m} \approx 0$. Another set or runs labelled $\mathrm{ABC}$ consists of a parameterized large scale helical flow, an uncorrelated and helical large scale magnetic field, and added noise with energy spectra $\sim k^{-3} \exp \left[-2\left(k / k_{0}\right)\right]^{2}$ at $t=0$, with $k_{0}=N / 6$ [10]. These runs have $E_{v}=E_{b}=0.5, \sigma_{c} \approx 1 \times 10^{-4}$, and $\sigma_{m} \approx 0.5$, while numerical resolution and Reynolds numbers vary (see Table I). Finally we analyze a small spatial region near a current sheet in a very high Reynolds number ABC simulation, ABC4 in the table.

Probability density functions. Our main diagnostics are probability density functions (pdfs) of the local cosine of the angle $\theta$ between $\mathbf{v}$ and $\mathbf{b}$

$$
\cos \theta=\cos (\mathbf{v}, \mathbf{b})=\frac{\mathbf{v} \cdot \mathbf{b}}{|\mathbf{v}||\mathbf{b}|}
$$

which are computed for each run.

The distribution function for RAN2 is shown Fig(1), at times $t=0,0.5,1.0$ and 2.0. These distributions are highly peaked near $\cos \theta \approx 1$, much more so than would be needed to account for the cross helicity which is initially $\sigma_{c} \approx 0.5$, decaying to $\sigma_{c}=0.24$ at $t=2.0$. The more peaked curves are for the progressively later times. The results for RAN1, having no helicities, are shown in Fig. 2. Now, the distributions are suppressed near $\cos \theta \approx 0$ and strongly peaked near $\cos \theta \approx \pm 1$ indicating an enhanced probability of magnetic and velocity field being very nearly aligned or antialigned. Enhanced directional alignment occurs even when the globally averaged cross helicity is approximately zero. We do not show results for RAN3 and RAN4, with $\sigma_{m} \approx 0.5$, as the distributions are almost indistinguishable from the corresponding case with $\sigma_{m} \approx 0$.

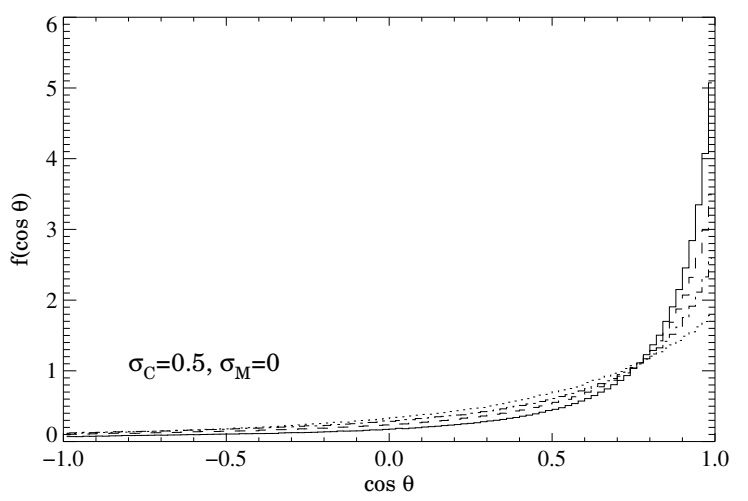

FIG. 1: Pdfs of $\cos \theta$ for initial normalized cross helicity $\sigma_{c}=$ 0.5 for Run RAN2. Global normalized cross helicity is 0.24 at $\mathrm{t}=2$. Different lines are for different times (see text).

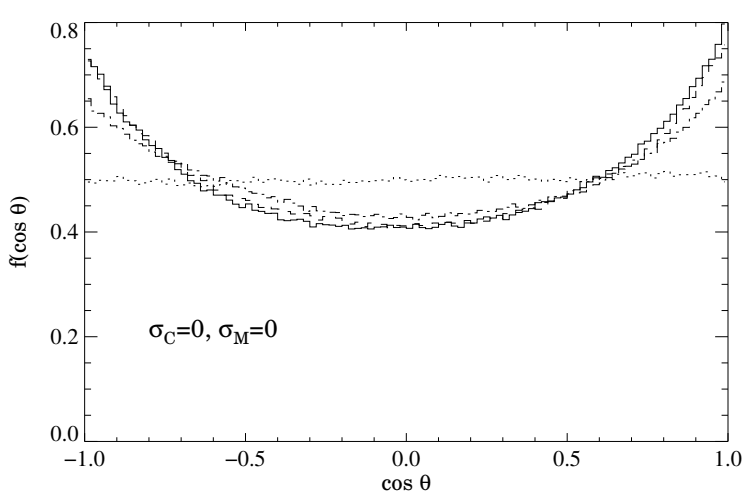

FIG. 2: Pdfs of $\cos \theta$ at times $\mathrm{t}=0$ (dotted), 0.5 (dash-dotted), 1.0 (dashed), 2 (solid) in a $3 \mathrm{D}$ simulation $\sigma_{c} \approx 0$ and $\sigma_{m} \approx 0$ (Run RAN1). The initial distribution is flat.

The pdfs in the OT runs (not shown) are asymmetric and strongly peaked at $\cos \theta \approx 1$, as in the RAN2 and RAN4 cases. For the ABC runs, with no net crosshelicity, the pdfs peak at $\cos \theta \approx \pm 1$ after less than half a turnover time, following the pattern of the RAN runs. Figure 3 shows the pdfs from the ABC runs at the peak of dissipation $(t \approx 4)$ for different Reynolds numbers.

This local alignment process is fast in all cases, with substantial and apparently nearly saturated alignment occurring in less than one large scale turnover time. As stated above, no clear dependence with the Reynolds numbers is seen when we compare cases ABC1-4.

When pdfs of $\cos \theta$ are computed in the vicinity of a cluster of strong current sheets, or in regions of strong shear in the magnetic field (run ABC4), an only slightly different result is obtained (Fig. 4). Inside the current sheet, the magnetic and velocity field are strongly antialigned (which gives the peak near -1 ), and the pdf is linear. As larger subvolumes surrounding the current sheet are considered, or at later times when current 


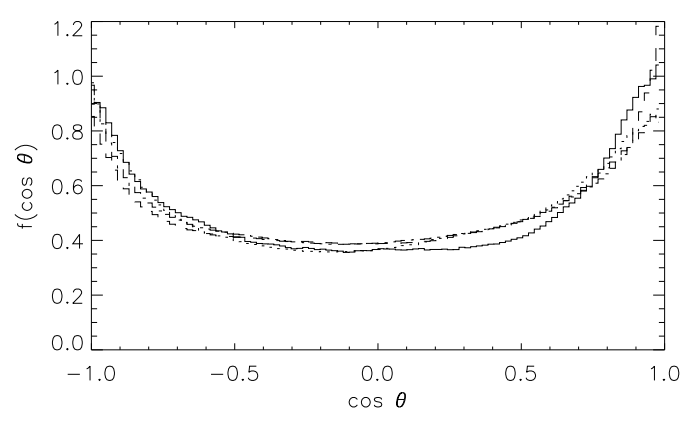

FIG. 3: Pdfs of $\cos \theta$ in runs $\mathrm{ABC} 1$ (solid), $\mathrm{ABC} 2$ (dotted), and $\mathrm{ABC} 3$ (dashed) at the peak of energy dissipation and ABC4 (dash-dotted).

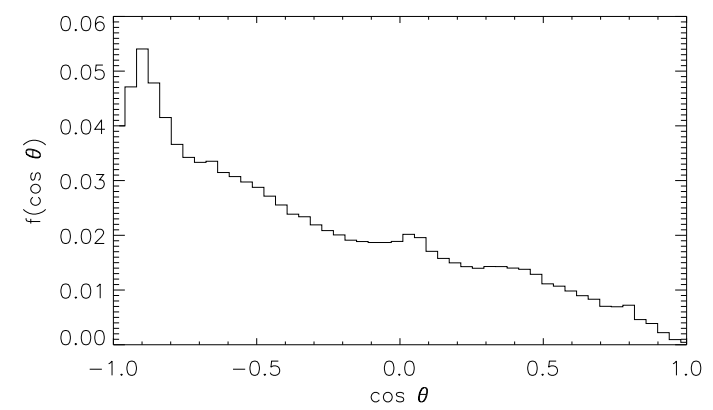

FIG. 4: Pdfs of $\cos \theta$ in the vicinity of a current sheet (subvolume of $150^{3}$ grid points) in a $1536^{3}$ simulation with $\mathrm{ABC}$ plus noise initial conditions (run $\mathrm{ABC} 4$ ).

sheets accumulate and interact, and thus more current sheets with different alignments are integrated in the subvolume, the pdf converges towards the form seen in Fig. (3) for the whole flow.

Physics of alignment. Why does local alignment take place in these simulations? And why is it so fast? Manipulating the MHD equations, Eqs. (1) and (2) in the ideal case $(\nu=\eta=0)$, one finds the equation for evolution of the local cross helicity:

$$
\frac{\partial(\mathbf{v} \cdot \mathbf{b})}{\partial t}+\mathbf{v} \cdot \nabla(\mathbf{v} \cdot \mathbf{b})=\mathbf{b} \cdot \nabla \frac{\mathbf{v}^{2}}{2}-\mathbf{b} \cdot \nabla \mathcal{P} .
$$

The terms on the left are the convective derivative, indicating that $\mathbf{v} \cdot \mathbf{b}$ is advected by the velocity field. The terms on the right are divergences: using that $\nabla \cdot \mathbf{b}=0$, and when integrated over volume with the proper boundary conditions (e.g. periodic boundaries) they vanish. This expresses the simple fact that the total cross helicity is an ideal invariant in MHD.

However, gradients of kinetic energy and pressure gradients affect the local alignment between the two fields. The first term on the right of Eq. (4) shows that gradients in the kinetic energy (e.g., shear) can change the alignment between the fields when they are parallel to the magnetic field lines. Indeed, a magnetic field line (which

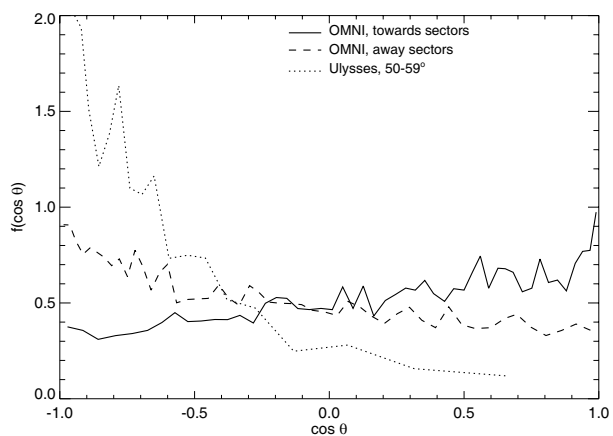

FIG. 5: Pdf of $\cos (\mathbf{v}, \mathbf{b})$ from 30 years of Omni data (ISEE, IMP and other satellite data). Also shown is the pdf of $\cos (\mathbf{v}, \mathbf{b})$ from Ulysses spacecraft data between 50 and $59^{\circ}$ heliospheric North latitude during a polar pass in solar minimum conditions.

behaves as a material line as follows from Alfvén's theorem) tends to be distorted by the shear, and aligned with the velocity field locally. For a planar shear, this would be very similar to what is called field-line stretching. Pressure gradients aligned along magnetic field lines, from the second term on the right, also change the alignment. Where a pressure gradient is present, velocity goes from the region of higher pressure to the region of lower pressure. If the pressure gradient has a projection onto the magnetic field, the resulting velocity field will be aligned as a result with the magnetic field. Moreover, from dimensional analysis we can estimate the time for the local alignment to take place as $\sim b_{l} / l$, where $b_{l}$ is the amplitude of the magnetic fluctuations at scale $l$.

Note that the induction equation is formally equivalent to the vorticity equation in hydrodynamics. Consequently by the same reasoning, it can be shown that the hydrodynamic velocity and vorticity fields tend to align locally, as found numerically in [7] for regions of low dissipation. This, replacing $\mathbf{b}$ by the vorticity $\boldsymbol{\omega}=\nabla \times \mathbf{v}$ in Eq. (4), occurs according to alignment of $\boldsymbol{\omega}$ with gradients of the kinetic energy and the pressure.

Solar wind observations. Using samples of spacecraft data we computed distributions of the alignment angle for two interplanetary datasets - the Omni dataset at 1AU near Earth orbit in the ecliptic plane, and a sample of Ulysses data from high heliographic latitude. Fig. (5) shows the results of these analyses. The low latitude OMNI analysis is divided into intervals in which the large scale interplanetary magnetic field is directed either away from or towards the sun. The net Alfvénicity is outward at the higher latitude of the Ulysses sample. In each of these cases, the pdfs of the local alignment are consistent with the net cross helicity in each sample.

Discussion and conclusions. The characteristic pdfs of $\cos \theta$ described above cannot be explained as a superposition of two uncorrelated Gaussian distributions for the velocity and magnetic fields, although the pdfs of the velocity and magnetic field themselves are Gaus- 


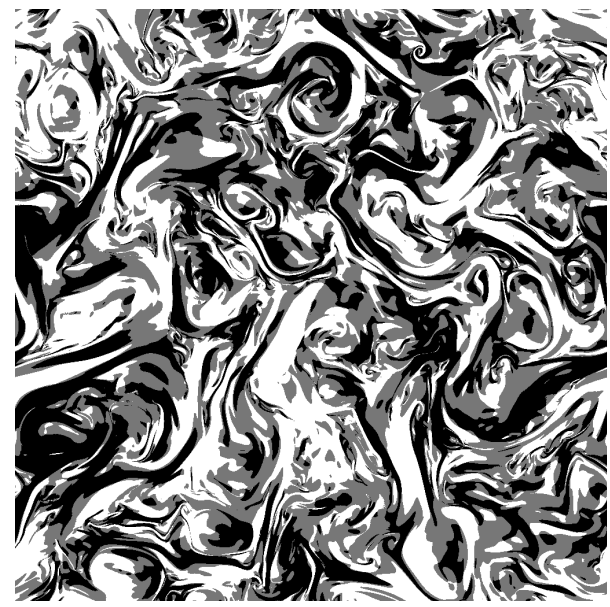

FIG. 6: Cross-helicity density in a 2D incompressible MHD simulation, showing areas that have values of $\cos (\mathbf{v}, \mathbf{b})<-0.7$ (black), $|\cos (\mathbf{v}, \mathbf{b})|<0.7$ (gray) and $\cos (\mathbf{v}, \mathbf{b})>0.7$ (white). Areas having highly aligned or anti-aligned velocity and magnetic field fluctuations dominate the picture.

sian (but clearly correlated). Pdfs computed from random broadband uncorrelated Gaussian-component velocity and magnetic fields have a flat $f(\cos \theta)$ distribution. For the coherent $\mathrm{ABC}$ flows, $\cos \theta$ peaks at 0 initially, while for the non-helical RAN1 and RAN3 flows the distribution is initially flat. All cases evolve towards the characteristic shape that is high-peaked at $|\cos \theta|=1$. In contrast, prior studies have shown that the distribution of the induced emf, $\mathbf{v} \times \mathbf{b}$, is accurately computed from the Gaussian statistics, for both high and low cross helicity, in simulations and in solar wind data [11]. What apparently accounts for the difference is that the induced emf does not correspond to a conserved quantity, while the alignment angle is closely associated with the ideally conserved cross helicity. The emf can be accounted for using Gaussian statistics, but alignment, even of Gaussian fields, is a dynamical quantity constrained by the local transport and conservation, as implied by Eq. (4).

Note that Alfvén vortices [12], which are coherent structures predicted for reduced MHD, have been recently observed in space plasmas [13]; the generalized Alfvén condition obeyed by these vortices corresponds to a local directional alignment. Evidently this type of robust alignment process may be influential in a variety of space and astrophysical plasmas in which turbulent relaxation operates, as well as in the neutral fluid case.

We conclude that directional alignment is a rapid and robust process in turbulence. The magnetic and velocity fields respond, as described above, according to the local values of the shear and pressure gradients, essentially independently of the conditions at remote locations, leading to local alignment or anti-alignment; Fig. 6]illustrates this localization or patchiness of the directional alignment, using a 2D MHD simulation (see also [14]). Since the alignment appears to be an essentially universal and rapid process, it would not be surprising if the coherent small scale structures in turbulence are associated with it. Indeed, the case shown in Fig. 4 is such an example where current sheets are observed to have maximum alignment between the velocity and the magnetic fields 10]; similarly the local $\mathbf{v}-\boldsymbol{\omega}$ alignment may explain the slow return to full isotropy in fluid turbulence.

Research supported in part by NSF under ATM0539995 and by NASA under NASA NNG06GD47G. Computer time was provided in part by NCAR. PDM is a member of the Carrera del Investigador Científico of CONICET.
[1] L. Woltjer. Proc. Nat. Acad. Sci. USA, 44:833, 1958.

[2] J. W. Belcher and L. Davis Jr. J. Geophys. Res., 76:3534, 1971.

[3] B. Bavassano, M. Dobrowolny, G. Fanfoni, F. Mariani, and N. F. Ness. Solar Phys., 78:373, 1982; D. A. Roberts, M. L. Goldstein, L. W. Klein and W. H. Matthaeus, J. Geophys. Res. 92, 12023 (1987).

[4] M. Dobrowolny, A. Mangeney, and P. Veltri. Phys. Rev. Lett., 45:144, 1980; R. Grappin, U. Frisch, J. Léorat, and A. Pouquet. Astron. Astrophys., 105:6, 1982; W. H. Matthaeus, M. L. Goldsteinn, and D. Montgomery, Phys. Rev. Lett., 51:1484, 1983.

[5] A. Pouquet, U. Frisch, and M. Meneguzzi. Phys. Rev. A, 33:4266, 1986.

[6] A. C. Ting, W. H. Matthaeus, and D. Montgomery. Phys. Fluids, 29:3261, 1986; T. Stribling and W. H. Matthaeus. Phys. Fluids B, 3:1848, 1991.

[7] R. Pelz et al. Phys. Rev. Lett. 54:2505, 1985; H.K. Moffatt, J. Fluid Mech. 150, 359 (1985); A. Tsinober and E. Levich, Phys. Lett. 99A, 321 (1983); E. Levich, Phys. Rep. 151, 129 (1987); M. Farge, G. Pellegrino, and K.
Schneider, Phys. Rev. Lett. 87, 054501 (2001)

[8] S. A. Orszag and G. S. Patterson. Phys. Rev. Lett., 28:76, 1972.

[9] S. A. Orszag and C-M Tang. J. Fluid Mech., 90:129, 1979.

[10] P. Mininni, A. Pouquet and D. Montgomery, Phys. Rev. Lett. 97, 244503, 2006; P. Mininni and A. Pouquet, preprint (2007), see http://arxiv.org/abs/0707.3620v1.

[11] B. Breech, W. H. Matthaeus, L. J. Milano, and C. W. Smith. J. Geophys. Res., 108:1153, doi:1029/2002JA009529, 2003.

[12] V.I. Petviashvili and O. A. Pokhotelov, in Solitary Waves in Plasmas and in the Atmosphere, Gordon and Breach, New York (1992).

[13] D. Sundkvist et al., Nature (London) , 436:825, 2005; O. Alexandrova et al., J. Geophys. Res., 111:A12208, doi:10.1029/ 2006JA011934, 2006.

[14] M. Meneguzzi, H. Politano, A. Pouquet and M. Zolver, J. Comp. Phys. 123, 32, 1996. 